\title{
Incurvación anterolateral congénita de la tibia asociada con polidactilia. Reporte de un caso y revisión bibliográfica
}

\author{
Paula Biolatto, Miguel Carabajal Mattar, Florencia Turazza, Julio J. Masquijo \\ Departamento de Ortopedia y Traumatología Infantil, Sanatorio Allende, Córdoba, Argentina
}

\begin{abstract}
RESUMEN
La incurvación anterolateral congénita de la tibia asociada con polidactilia (congenital anterolateral tibial bowing associated with polydactyly) es una deformidad extremadamente rara. Solo se han documentado 21 casos en la bibliografía inglesa. Aunque existe una estrecha relación entre la incurvación anterolateral de la tibia, la seudoartrosis congénita de la tibia y la neurofibromatosis tipo 1, la incurvación anterolateral congénita de la tibia asociada con polidactilia es una entidad específica con un pronóstico más favorable. Comunicamos otro caso y también revisamos los casos de este cuadro ya reportados con respecto a las deformidades asociadas, el diagnóstico y el tratamiento.
\end{abstract}

Palabras clave: Incurvación tibial; polidactilia; hallux.

Nivel de Evidencia: IV

Congenital anterolateral tibial bowing associated with polydactyly: case report and literature review

\section{ABSTRACT}

The congenital anterolateral tibial bowing associated with polydactyly (CABTP) is an extremely rare deformity. To the best of our knowledge, only 21 cases have been documented in the English literature. Although there is a strong relationship between anterolateral bowing of the tibia, congenital pseudoarthrosis of the tibia (CPT), and neurofibromatosis type 1 (NF1), CATBP is a specific entity associated with a more favorable prognosis. We report a new CABTP case and our literature review on the previously reported cases of CABTP concerning its associated deformities, diagnosis, and treatment.

Key words: Tibial bowing; polydactyly; hallux.

Level of Evidence: IV

\section{INTRODUCCIÓN}

La incurvación congénita de la tibia es un cuadro ortopédico poco frecuente. El ápex de la deformidad puede presentarse en sentido anterolateral, anteromedial o posteromedial. ${ }^{1}$ Cada tipo de deformidad suele estar asociada a distintas etiologías. La incurvación anteromedial se asocia con la hemimelia peronea. Esta condición representa la deficiencia más común de los huesos largos y suele manifestarse con un amplio espectro de deformidades, que varían desde una leve discrepancia de longitud de los miembros hasta un acortamiento severo del lado afectado con deformidades del pie, el tobillo y la rodilla. ${ }^{2}$ En la incurvación posteromedial, es constante la deformidad en talo del pie que remite espontáneamente en los primeros meses. Por lo general, también se puede producir la corrección espontánea, pero incompleta, de la deformidad tibial dentro de los primeros cuatro años de vida, aunque la discrepancia de miembros inferiores suele requerir tratamiento. ${ }^{3}$

La incurvación anterolateral es la forma con peor pronóstico por el riesgo de fractura patológica y seudoartrosis. ${ }^{4}$ Este tipo de incurvación está muy relacionada con la seudoartrosis congénita de la tibia (SCT) y la neurofibromatosis tipo 1. La evolución natural de la SCT es extremadamente desfavorable y, una vez que se produce una fractura, hay poca o ninguna tendencia a que la lesión se cure de manera espontánea. ${ }^{5}$ 
Si bien hay una estrecha relación entre la incurvación anterolateral de la tibia y la SCT, existe una forma de presentación extremadamente rara, de pronóstico más favorable, llamada incurvación anterolateral congénita de la tibia asociada con polidactilia (congenital anterolateral tibial bowing associated with polydactyly, CABTP). Se han documentado solo 21 casos. ${ }^{6-15}$

Comunicamos otro caso y analizamos las deformidades asociadas, el diagnóstico y el tratamiento de los casos ya reportados de CABTP.

\section{CASO CLÍNICO}

Varón de 6 meses de edad que fue derivado para la evaluación y el tratamiento de una polidactilia del pie izquierdo en el Departamento de Ortopedia y Traumatología Infantil de nuestra institución. El paciente no tenía antecedentes personales ni familiares destacables. El examen físico reveló polidactilia preaxial, hallux izquierdo con duplicación completa de la falange y el metatarsiano. El dedo supernumerario medial era hipoplásico y el espacio interdigital se encontraba ampliado (hallux varus) (Figura 1A).

A
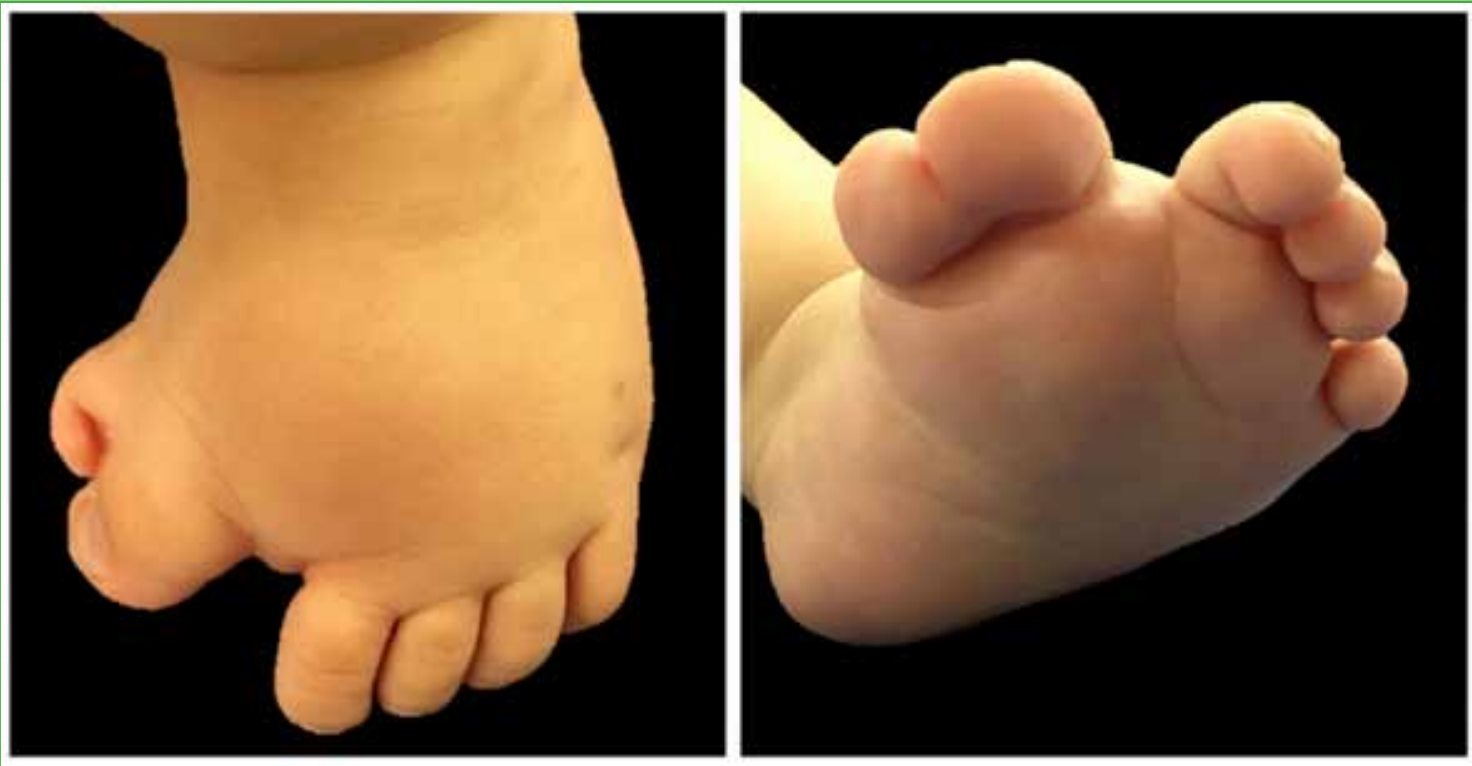

Figura 1. Varón de 6 meses. A. Imagen clínica. Se observa un hallux varus asociado a polidactilia preaxial.

Además, tenía una incurvación anterolateral de la tibia, con mínima discrepancia de longitud de los miembros inferiores. No se detectaron manchas "café con leche" en la piel ni otros signos de neurofibromatosis. Se solicitaron radiografías de ambos pies y miembros inferiores, en las que se confirmó la deformidad (Figura 1B y C). Debido a la asociación de deformidades se realizó una consulta genética que confirmó el diagnóstico de CABTP.

Luego de analizar el diagnóstico y el pronóstico de la enfermedad con los padres, se decidió efectuar el tratamiento quirúrgico de la deformidad del pie. Se resecó el dedo supernumerario y se realineó el hallux con la técnica de Farmer para disminuir el espacio interdigital. No hubo complicaciones durante la cirugía ni en el posoperatorio. A los 22 meses, el paciente tiene una buena evolución con una correcta alineación del hallux. La deformidad tibial evolucionó favorablemente, aunque la discrepancia de longitud se incrementó (Figuras 2 y 3). En la actualidad, utiliza realce en el calzado y requerirá cirugía para nivelar los miembros inferiores. 

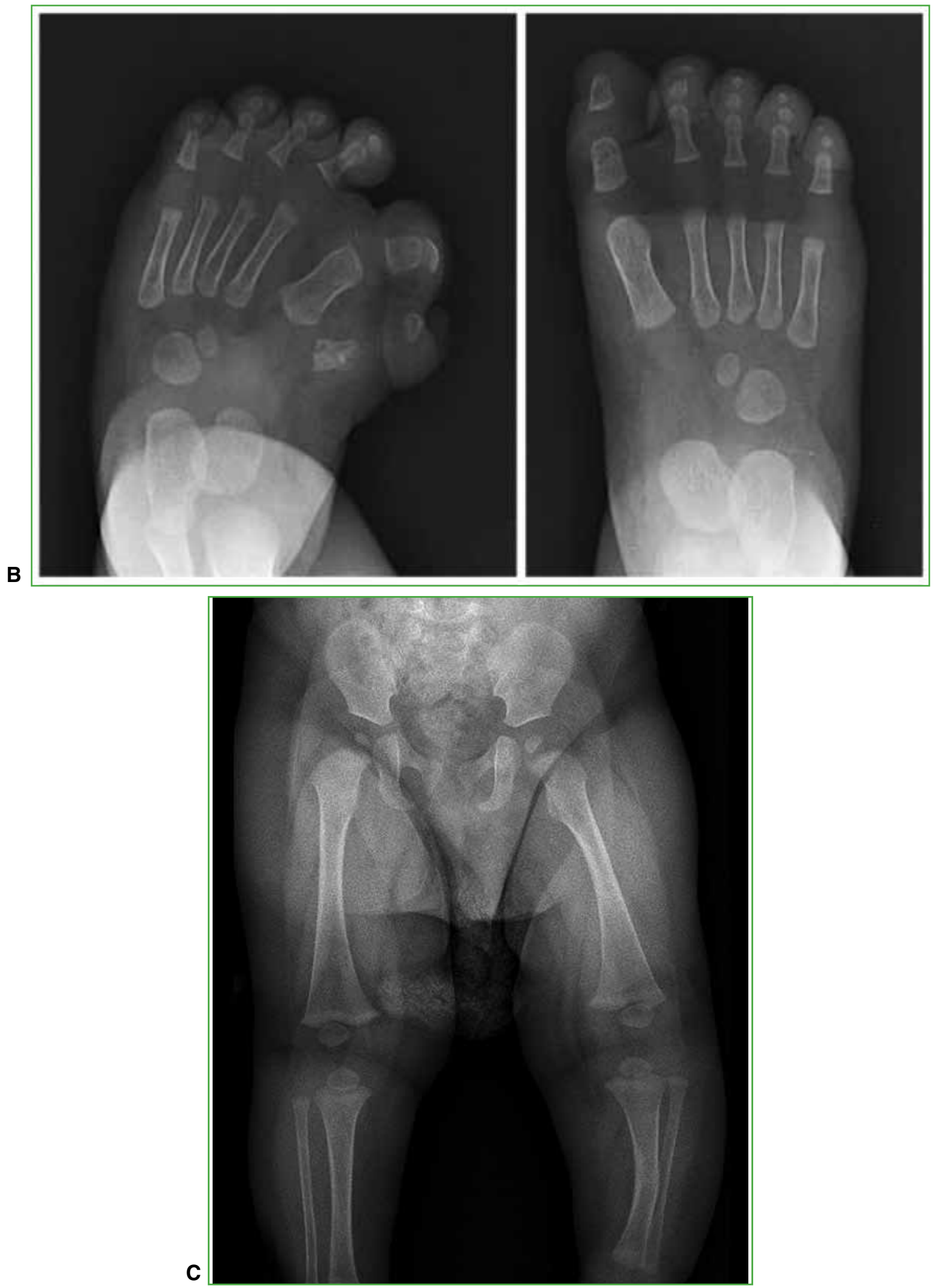

Figura 1. Varón de 6 meses. B. Radiografía de ambos pies, de frente que confirma el diagnóstico de polidactilia preaxial. C. Radiografía de miembros inferiores con incurvación anterolateral de la tibia izquierda y leve discrepancia de longitud de los miembros. 

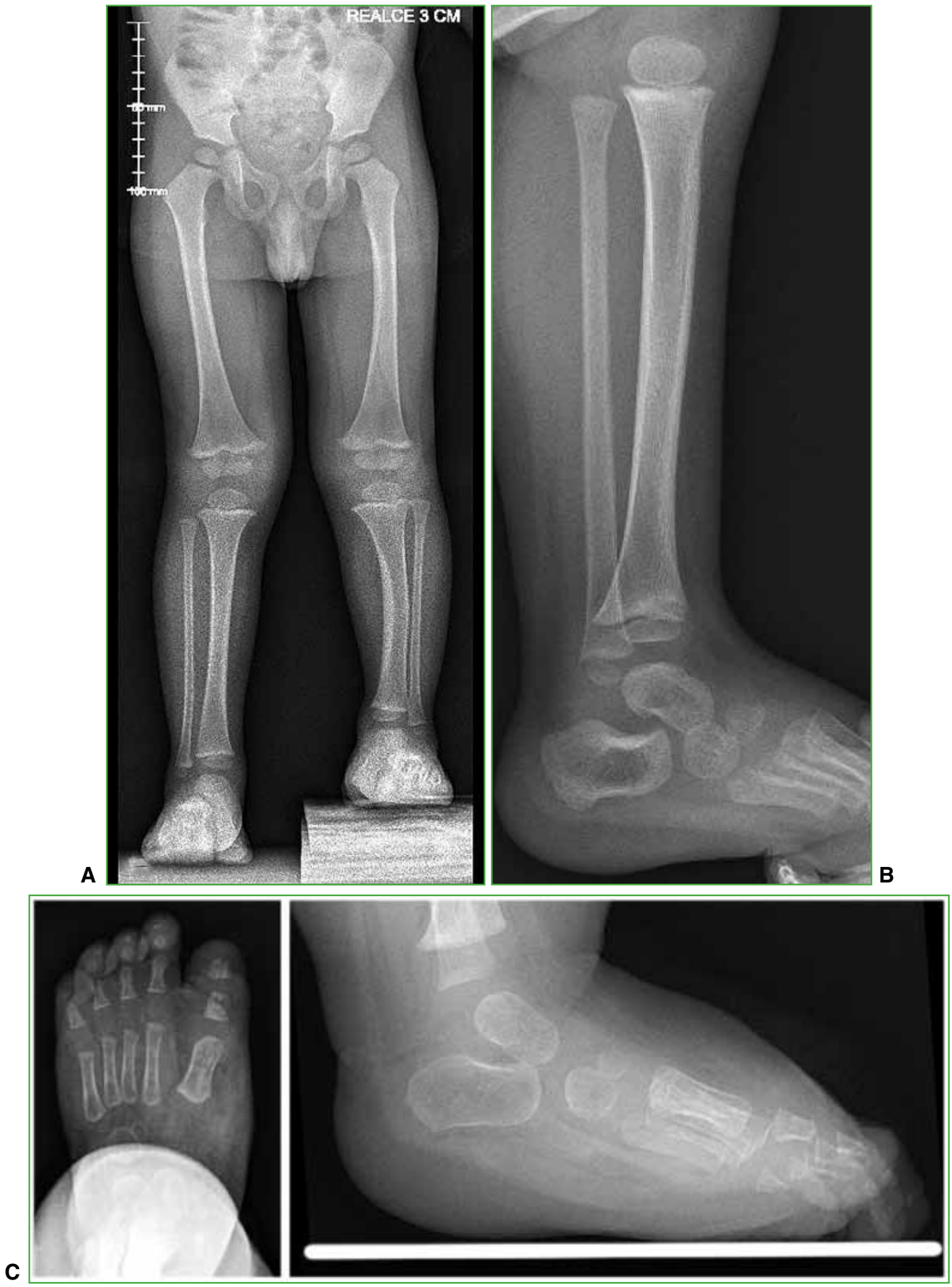

Figura 2. Paciente de la Figura 1, a los 2 años y 2 meses. A. Radiografía de miembros inferiores. Se observa una marcada mejoría de la incurvación anterolateral de la tibia izquierda y mayor discrepancia de longitud de miembros $(2,8 \mathrm{~cm})$. B. Radiografía de perfil de la tibia izquierda sin deformidad en el plano sagital. C. Radiografías del pie izquierdo, de frente y de perfil, a los 20 meses de la corrección de la polidactilia. 

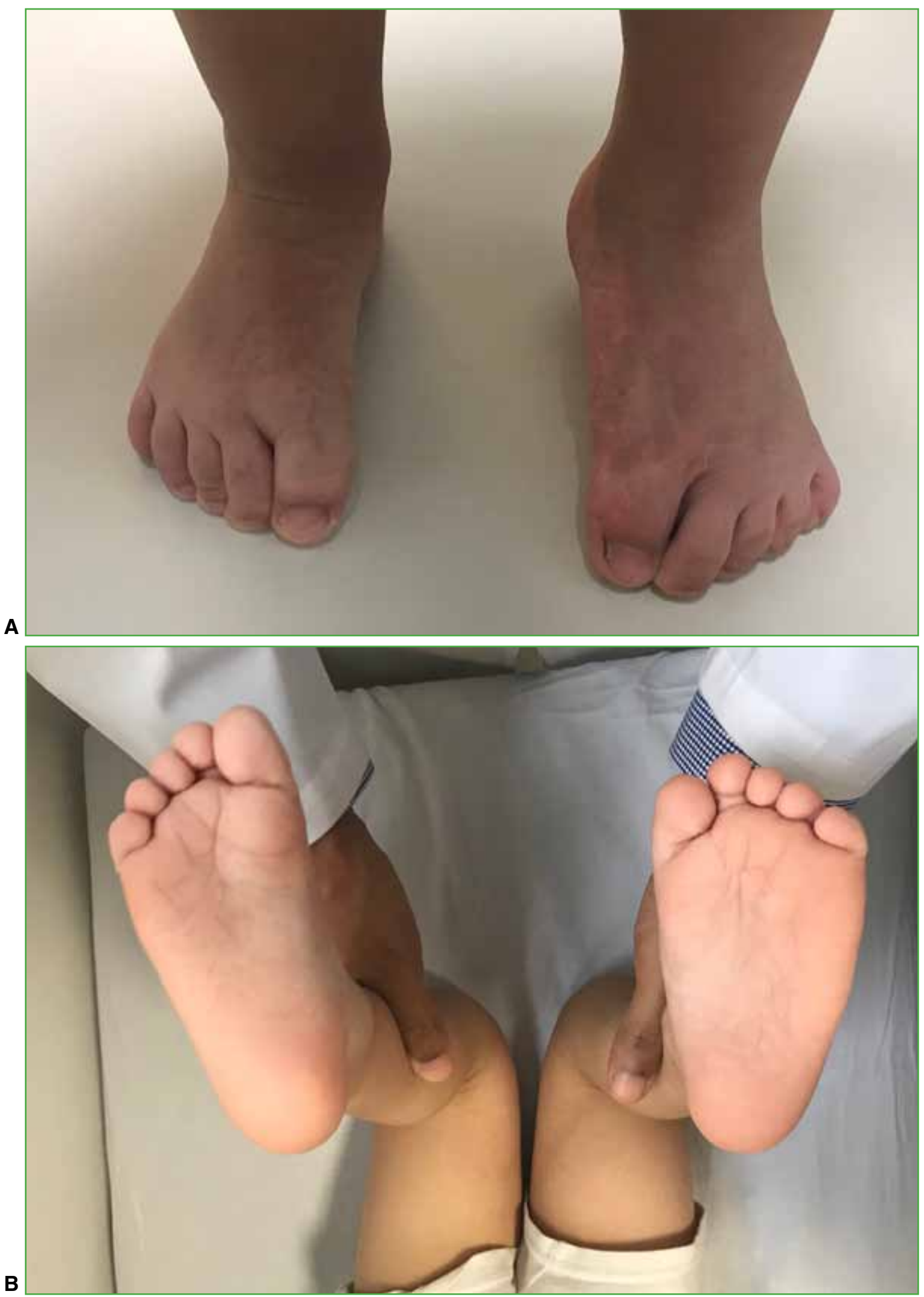

Figura 3. Imágenes clínicas posoperatorias. Obsérvense el menor tamaño del pie izquierdo (A y B). 

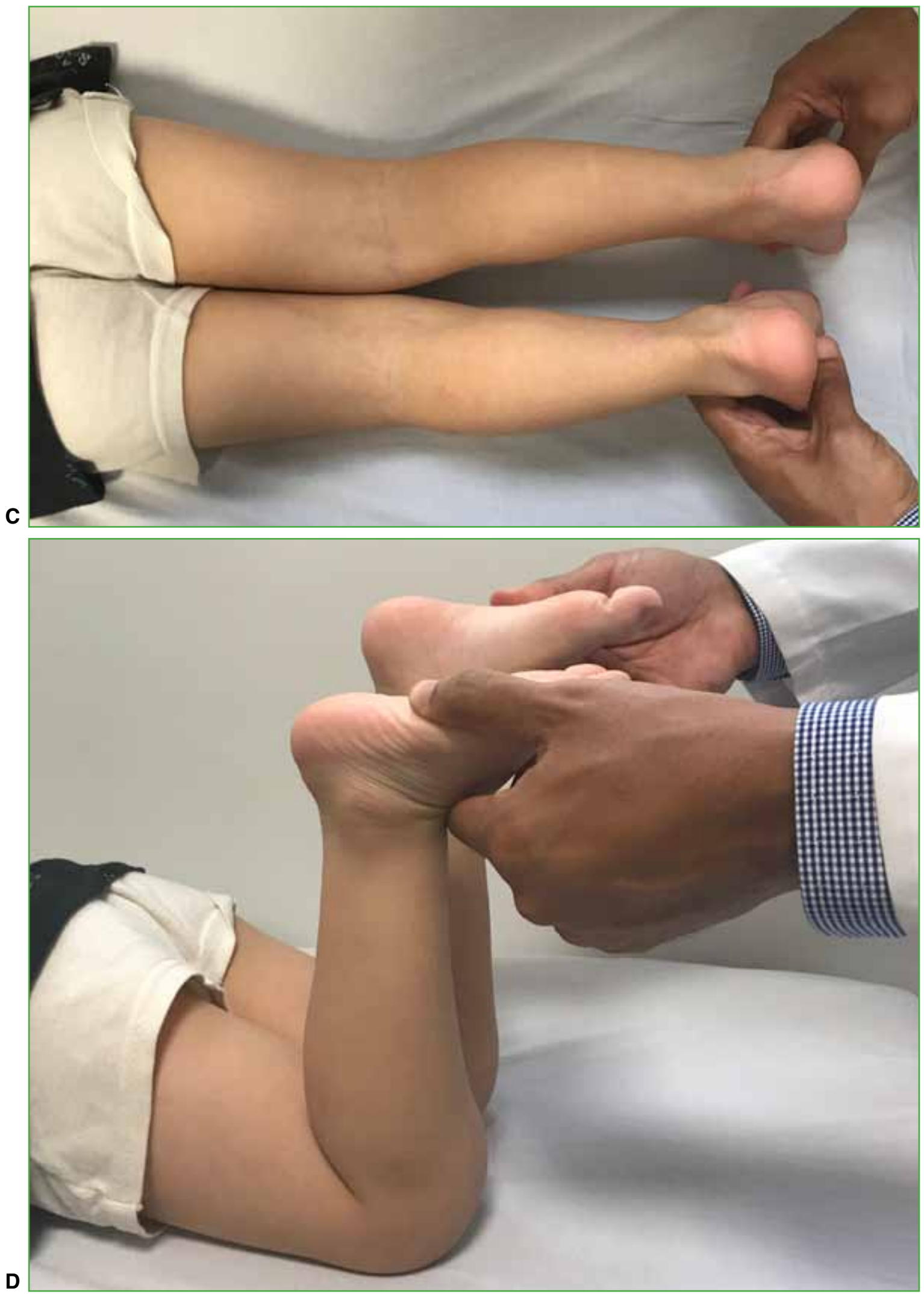

Figura 3. Imágenes clínicas posoperatorias. Discrepancia de longitud de los miembros inferiores (C y D). 


\section{DISCUSIÓN}

La incurvación congénita anterolateral de la tibia es una deformidad esquelética que, con frecuencia, se relaciona con la neurofibromatosis tipo $1 \mathrm{y}$ la SCT. ${ }^{1}$ La SCT es un cuadro desafiante, ya que la deformidad no se corrige espontáneamente y es difícil lograr la consolidación una vez instaurada la seudoartrosis. Por esta razón, el tratamiento inicial está dirigido a prevenir la deformidad progresiva y la fractura mediante el uso de ortesis. ${ }^{16}$ Aunque está muy relacionada, la incurvación anterolateral de la tibia no es exclusiva de la SCT y la neurofibromatosis tipo 1. Existe una variante que se asocia con polidactilia homolateral del hallux y que tiene un pronóstico más favorable. La CABTP es un cuadro de etiología desconocida, cuyo diagnóstico se basa en los hallazgos clínicos y radiográficos. Existen muy pocos casos publicados de esta entidad (Tabla).

Tabla. Características demográficas de los pacientes publicados con incurvación anterolateral congénita de la tibia asociada con polidactilia

\begin{tabular}{|c|c|c|c|c|c|c|}
\hline Autor & Publicación & n & Edad $^{*}$ & Sexo & Lado & Deformidades asociadas \\
\hline Newell & J Bone Joint Surg (1976) & 1 & 3 años & M & I & \\
\hline \multirow[t]{5}{*}{ Adamsbaum } & \multirow[t]{5}{*}{ Pediatr Radiol (1991) } & \multirow[t]{5}{*}{5} & 17 meses & $\mathrm{F}$ & I & $\begin{array}{l}\text { Sindactilia bilateral, duplicación del } \\
\text { pulgar }\end{array}$ \\
\hline & & & 12 años & M & $\mathrm{D}$ & Clinodactilia bilateral \\
\hline & & & 10 años & M & I & Clinodactilia izquierda \\
\hline & & & 7 meses & M & $\mathrm{D}$ & $\begin{array}{l}\text { Pie equinovaro, clinodactilia derecha, } \\
\text { hipertrofia de pulgar }\end{array}$ \\
\hline & & & 6 años & M & $\mathrm{D}$ & \\
\hline \multirow[t]{2}{*}{ Weaver } & \multirow[t]{2}{*}{ J Pediatr Orthop (1996) } & \multirow[t]{2}{*}{2} & 9 meses & M & I & \\
\hline & & & 6 años & M & I & $\begin{array}{l}\text { Duplicación de huesos navicular, } \\
\text { cuneiforme y metatarsiano, desviación } \\
\text { radial de ambos índices (mano) }\end{array}$ \\
\hline \multirow[t]{2}{*}{ Kitoh } & \multirow[t]{2}{*}{ Am J Med Genet (1997) } & 1 & 13 años & M & $\mathrm{D}$ & \\
\hline & & & 18 meses & & $\mathrm{D}$ & \\
\hline Bressers & J Pediatr Orthop B (2001) & 1 & 17 meses & M & I & \\
\hline \multirow[t]{3}{*}{ Manner } & \multirow[t]{3}{*}{ J Bone Joint Surg Br (2005) } & \multirow[t]{3}{*}{3} & 6 años & $\mathrm{F}$ & - & \\
\hline & & & 5 años & M & - & \\
\hline & & & 5 años & $\mathrm{F}$ & $\mathrm{D}$ & \\
\hline Lemire & J Med Case Rep (2007) & 1 & 2 años & M & $\mathrm{D}$ & Desviación radial, índice izquierdo \\
\hline \multirow[t]{2}{*}{ Breckpot } & \multirow[t]{2}{*}{ Clin Dysmorphol (2009) } & \multirow[t]{2}{*}{2} & 19 años & M & $\mathrm{D}$ & $\begin{array}{l}\text { Agenesia de cuerpo calloso, quiste } \\
\text { interhemisférico, } \\
\text { sindactilia simple }\end{array}$ \\
\hline & & & 2 años & M & $\mathrm{D}$ & $\begin{array}{l}\text { Agenesia de cuerpo calloso, quiste } \\
\text { interhemisférico, estenosis del acueducto, } \\
\text { clinodactilia derecha, hipoplasia distal de } \\
\text { índices }\end{array}$ \\
\hline \multirow[t]{3}{*}{ Han } & \multirow[t]{3}{*}{ Am J Med Genet Am (2012) } & \multirow[t]{3}{*}{3} & 28 meses & M & $\mathrm{D}$ & \\
\hline & & & 2 años & $\mathrm{M}$ & I & Incurvación leve del peroné \\
\hline & & & 2 años & M & I & \\
\hline \multirow[t]{2}{*}{ Beck } & \multirow[t]{2}{*}{ J Pediatr Orthop B (2013) } & \multirow[t]{2}{*}{2} & 15 años & M & I & Pie bot \\
\hline & & & 16 años & M & $\mathrm{D}$ & Pie bot \\
\hline Nuestro caso & & 1 & 2 años & M & I & \\
\hline
\end{tabular}

$\mathrm{M}=$ masculino, $\mathrm{F}=$ femenino, $\mathrm{I}=$ izquierdo, $\mathrm{D}=$ derecho, $\mathrm{NR}=$ no reportado.

*Edad en el momento del último control. 
En 1976, Newell y Durbin ${ }^{6}$ describieron un tipo de incurvación anterolateral de la tibia no relacionada con la neurofibromatosis tipo 1, pero sí con malformaciones congénitas, que tenía tendencia a la resolución espontánea. En 1991, Adamsbaum y cols. ${ }^{7}$ establecieron la relación entre incurvación anterolateral de la tibia y duplicación preaxial del hallux homolateral como una entidad separada, de mejor pronóstico. Más adelante, Weaver y cols., ${ }^{8}$ Bressers y Castelein, ${ }^{10}$ y Manner y cols. ${ }^{11}$ publicaron casos similares, con una descripción más detallada sobre los cambios morfológicos de la tibia mediante estudios radiográficos y tomografía.

Los pacientes con CABTP suelen tener otras deformidades asociadas en el miembro superior, como clinodactilia o polidactilia, y cerebrales, como agenesia del cuerpo calloso. Si bien la relación con otras deformidades de la mano o cerebrales no posee un patrón homogéneo, todos los pacientes tenían una incurvación tibial anterolateral unilateral y deformidad severa en varo del hallux con duplicación de falanges, primer metatarsiano o incluso huesos del tarso y siempre de presentación homolateral. ${ }^{6-14}$ Un aspecto distintivo en estos pacientes es que el ápex de la deformidad suele estar localizado en el tercio medio de la tibia a diferencia de la seudoartrosis donde el ápex suele estar más distal en la unión del tercio medio con el tercio distal. La morfología en el punto de incurvación de la tibia es variable, según los hallazgos tomográficos. Adamsbaum y cols. ${ }^{7}$ y Manner y cols. ${ }^{11}$ describieron una duplicación del canal medular tibial, mientras que Kitoh y cols. ${ }^{9}$ comunicaron la presencia de una hendidura en la cortical posteromedial de la deformidad. Los pacientes con esta enfermedad no suelen tener signos radiográficos de esclerosis, estrechamiento del canal y falta de consolidación, característicos en la seudoartrosis. Por otra parte, tampoco es común el compromiso del peroné, aunque sí puede tener una leve discrepancia o incurvación al compararlo con el contralateral. En todos los casos publicados, la resolución de la incurvación tibial fue espontánea. La deformidad que requiere tratamiento inicial es la duplicación del hallux (que suele ser tratada en los primeros meses de vida) y posteriormente la discrepancia de miembros inferiores.

En nuestro caso, el paciente no tenía antecedentes hereditarios ni otras malformaciones asociadas, tampoco compromiso del peroné. El seguimiento radiográfico mostró una buena evolución con corrección de la deformidad tibial, pero con progresión de la discrepancia. No consideramos necesario el estudio tomográfico a causa de la radiación, porque los hallazgos no cambian el tratamiento de la enfermedad. La cirugía precoz de la polidactilia preaxial permitió realinear el antepié y que el paciente utilizara calzado convencional sin dificultades. La discrepancia ha sido manejada inicialmente con realce, pero requerirá cirugía para igualar los miembros.

\section{CONCLUSIÓN}

La CABTP es un cuadro de etiología desconocida que incluye incurvación anterolateral de la tibia unilateral asociada a duplicación del hallux homolateral. El diagnóstico es clínico y radiográfico. La deformidad tibial se localiza en la región mediodiafisaria (más proximal que en la SCT) y tiende a la corrección espontánea. Esta distinción permite evitar tratamientos ortésicos innecesarios y aconsejar a la familia sobre el pronóstico más favorable de esta enfermedad.

Conflicto de intereses: Los autores no declaran conflictos de intereses.

ORCID de M. Carabajal Mattar: https://orcid.org/0000-0002-0301-0867

ORCID de F. Turazza: hitps://orcid. org/0000-0002-8705-0304

ORCID de J. J. Masquijo: https://orcid.org/0000-0001-9018-0612

\section{BIBLIOGRAFÍA}

1. Hefti F, Bollini G, Dungl P, Fixsen J, Grill F, Ippolito E, et al. Congenital pseudarthrosis of the tibia: history, etiology, classification, and epidemiologic data. J Pediatr Orthop Br 2000;9(1):11-5. https://doi.org/10.1097/01202412-200001000-00003

2. Crawford DA, Tompkins BJ, Baird GO, Caskey PM. The long-term function of the knee in patients with fibular hemimelia and anterior cruciate ligament deficiency. J Bone Joint Surg Br 2012;94(3):328-33. https://doi.org/10.1302/0301-620X.94B3.27997 
3. Shah HH, Doddabasappa SN, Joseph BJ. Congenital posteromedial bowing of the tibia: a retrospective analysis of growth abnormalities in the leg. J Pediatr Orthop Br 2009;18(3):120-8. https://doi.org/10.1097/BPB.0b013e328329dc86

4. Currarino G, Herring JA, Johnston CE Jr, Birch JG. An unusual form of congenital anterolateral tibial angulationthe delta tibia. Pediatr Radiol 2003;33(5):346-53. https://doi.org/10.1007/s00247-002-0856-7

5. Gilbert A, Brockman R. Congenital pseudarthrosis of the tibia. Long-term follow-up of 29 cases treated by microvascular bone transfer. Clin Orthop Relat Res 1995;314:37-44. PMID: 7634649

6. Newell RL, Durbin FC. The aetiology of congenital angulation of tubular bones with constriction of the medullary canal, and its relationship to congenital pseudarthrosis. J Bone Joint Surg Br 1976;58:444-7. PMID: 1018030

7. Adamsbaum C, Kalifa G, Seringe R, Bonnet JC. Minor tibial duplication: a new cause of congenital bowing of the tibia. Pediatr Radiol 1991;21(3):185-8. https://doi.org/10.1007/BF02011043

8. Weaver KM, Henry GW, Reinker KA. Unilateral duplication of the great toe with anterolateral tibial bowing. $J$ Pediatr Orthop 1996;16:73-7. https://doi.org/10.1097/00004694-199601000-00015

9. Kitoh H, Nogami H, Hattori T. Congenital anterolateral bowing of the tibia with ipsilateral polydactyly of the great toe. Am J Med Genet 1997;73:404-7. PMID: 9415465

10. Bressers MM, Castelein RM. Anterolateral tibial bowing and duplication of the hallux: a rare but distinct entity with good prognosis. J Pediatr Orthop Br 2001;10(2):153-7. PMID: 11360782

11. Manner HM, Radler C, Ganger R, Grossbotzl G, Petje G, Grill F. Pathomorphology and treatment of congenital anterolateral bowing of the tibia associated with duplication of the hallux. J Bone Joint Surg Br 2005;87:226-30. https://doi.org/10.1302/0301-620x.87b2.15132

12. Lemire EG. Congenital anterolateral tibial bowing and polydactyly: a case report. J Med Case Rep 2007;1:54. https://doi.org/10.1186/1752-1947-1-54

13. Breckpot J, Thienpont B, Vanhole C, Van Rossem E, Van Schoubroeck D, Fryns JP, et al. Congenital anterolateral bowing of the tibia with ipsilateral polydactyly of the hallux associated with cerebral cyst: A new entity? Clin Dysmorphol 2009;18:195-200. https://doi.org/10.1097/MCD.0b013e32832d06d7

14. Han J, Qu L, Li Y, Luo J, Cao J, Zhao W. A benign form of congenital anterolateral bowing of the tibia associated with ipsilateral polydactyly of the hallux: case report and literature review. Am J Med Genet Am 2012;158(7):17429. https://doi.org/10.1002/ajmg.a.35417

15. Beck JJ, Altiok H. Congenital tibial dysplasia with lateral bowing and duplication of hallux: case presentations. $J$ Pediatr Orthop B 2013;22(3):213-8. https://doi.org/10.1097/BPB.0b013e328352ad09

16. Jahmani R, Alorjani M. Anterolateral bowing of congenital pseudoarthrosis of tibia treated by percutaneous osteotomy and gradual correction using Taylor spatial frame, then late insertion of a Fussier-Duval nail: a case report. Am J Case Rep 2018;19:426-30. https://doi.org/10.12659/ajcr.908482 